\title{
METAPHORISCHE OBJEKTIVATION VON GENDERSTEREOTYPEN
}

\author{
Natalija Korolowa \\ Kandidat der philologischen Wissenschaften, Dozentin am Lehrstuhl für deutsche Philologie, \\ Fakultät für Fremdsprachen, Nationale Wassyl-Stefanyk Universität, Ukraine \\ e-mail: eiwissn@gmail.com,orcid.org/0000-0002-0093-7288

\section{Wojciech Żukowski} \\ Ph.D., Polonia University in Czestochowa, Interdisciplinary Faculty, Poland \\ e-mail: wzukowski@ap.edu.pl,orcid.org/0000-0002-8729-9708
}

\section{Zusammenfassung}

Der Artikel untersucht die genderspezifischen Merkmale der Verwendung von Metaphern zur Charakterisierung der handelnden Personen von Romanen "A Game of Thrones", "Die Herren von Winterfell" und "Das Erbe von Winterfell". Im Rahmen des Studiums eines Kunstwerks bilden genderspezifische Metaphern ein wichtiges Mittel der Verbalisierung und Aktualisierung von Charakterzügen und Verhaltensmerkmalen, die Frauen und Männern als handelnden Personen anhaften.

Durch den Vergleich der metaphorischen Nominationen von Frauen und Männern in der Originalsprache und in der Übersetzung konnte festgestellt werden, dass die deutsche Version die innere Form von Metaphern als bildliches Merkmal der metaphorischen Nomination einer Person beibehält. Darüber hinaus wurde auch Ähnlichkeit der Semantik von Nominationen der Vergleichsobjekte in beiden Sprachen festgestellt, d.h. nicht nur die bildliche, sondern auch die inhaltliche Ähnlichkeit der metaphorischen Namen von Frauen und Männern lässt sich zurückverfolgen. Dies liegt daran, dass die Wahl eines bestimmten Bildes nicht nur auf der subjektiven Intention des Autors der Metapher beruht, sondern auch auf seiner Weltanschauung und dem System stereotyper Bilder und Prototypen, die Bestandteile seines Weltbildes sind. Das Fehlen oder Vorhandensein eines gemeinsamen Bildes für bestimmte Sprachen ist auf den Einfluss der außersprachlichen Realität zurückzuführen. Durch das ethnische Bewusstsein beeinflusst sie die Sprache, modelliert die Interpretationsweisen der Realität und bildet bestimmte Parameter der Lebenssicht.

Wahrscheinlich, führte der gemeinsame deutsch-skandinavische mythologische Raum zur Entstehung von Bildern, die für beide Sprachen identisch sind und Männer und Frauen im Rahmen der untersuchten Werke charakterisieren.

Schlüsselwörter: Gender; genderspezifische Metapher; Genderstereotype; genderspezifische Unterschiede; Gendersemantik.

DOI: https://doi.org/10.23856/4005

\section{Einleitung}

Die letzten Jahrzehnte des XX. Jahrhunderts sind gekennzeichnet durch Bildung und intensive Entwicklung von Genderforschungen in der Linguistik, die mit dem Wechsel des wissenschaftlichen Paradigmas in den Geisteswissenschaften verbunden sind. Der neue Ansatz sah auch die Verwendung von neuer Terminologie vor, die besser für die methodischen Richtlinien von Wissenschaftlern geeignet war, und wurde zum Grund für die Einführung des Begriffs Gender in den wissenschaftlichen Diskurs, der die Konventionalität und Institutionalisierung 
des Begriffs Geschlecht widerspiegelte. Dieser Ansatz stimulierte die Erforschung der sprachlichen Mechanismen der Manifestation von Gender in Sprache und Kommunikation (Smith, 1997: 154-161). Im Bewusstsein von Vertretern verschiedener Kulturen werden die Kategorien Männlichkeit und Weiblichkeit nicht auf die gleiche Weise interpretiert und mit unterschiedlichen nationalen Ideen und Stereotypen in Verbindung gebracht, die sowohl die Position der Gesellschaft gegenüber Männern und Frauen als auch das Verhalten von Personen im Zusammenhang mit der Zugehörigkeit zu konkretem Geschlecht bestimmen, das sich in verschiedenen Arten von Diskursen widerspiegelt, einschließlich Kunstwerken.

In der Sprache spiegeln sich Genderstereotype auf der lexikalischen, phraseologischen und syntaktischen Ebene wider, einschließlich des Systems genderspezifischer Metaphern, die in der Genderlinguistik die Übertragung nicht nur physischen, sondern auch aller geistigen Qualitäten und Eigenschaften, die die Begriffe Weiblichkeit und Männlichkeit beinhalten, auf Objekte, die nicht geschlechtsbezogen sind, bedeuten (Kyrylyna, 2005: 7-32).

Nach S. I. Resanova betrachten wir als Gendermetaphern die metaphorischen Nominationen von Männern und Frauen, die als Mittel zur Kennzeichnung typisch männlicher und typisch weiblicher Eigenschaften auf der Grundlage der Angleichung von Phänomenen verschiedener Begriffsreihen dienen (Riezanova, 2011: 47-58).

Die ersten Versuche der Genderforschungen in der Linguistik gehen auf die 70-80er Jahre des XX. Jahrhunderts zurück. Die grundlegende Arbeit dieser Richtung war das Werk von R. Lakoff, in der die Autorin die anthropozentrische Ordnung der Sprache und eine gewisse Lückenhaftigkeit des Frauenbildes im sprachlichen Weltbild bestätigte (Andriichenko, 2011: 3-9). Linguisten schenken ihre Aufmerksamkeit Genderforschungen auf solchen Gebieten wie Kulturlinguistik, Psycholinguistik, Soziolinguistik, kognitive Linguistik, Konzeptologie, Ethnolinguistik, Diskursologie und kommunikative Linguistik auf der Grundlage von Kulturstudien genderspezifischer konzeptueller Inhalte von Wortschatz, Phraseologie, Parömien, kommunikativen Einheiten usw. (Semashko, 2010: 166-169).

Ziel unserer Studie besteht darin, die theoretischen Grundlagen von Genderfragen in der modernen Linguistik und genderspezifische Merkmale der Verwendung von Metaphern zu untersuchen, womit die Besonderheiten weiblicher und männlicher Figuren in Romanen charakterisiert werden können, sowie die metaphorischen Benennungen von Frauen und Männern in der Originalsprache und in der deutschen Übersetzung zu vergleichen. Forschungsobjekt bilden genderspezifische Metaphern, Forschungsgegenstad beruht auf Darstellungsarten von metaphorischen Benennungen von Frauen und Männern im Text von Romanen "A Game of Thrones", "Die Herren von Winterfell" und "Das Erbe von Winterfell". Forschungsmaterial stellen alle metaphorischen Personenbezeichnungen, die in den Texten von Romanen aktualisiert werden, zusammen.

\section{Genderspezifische Metaphern als Träger von Genderstereotypen}

Im Rahmen des Studiums eines Kunstwerks betrachten wir genderspezifische Metaphern als solche, die sowohl bestimmten Merkmale als auch genderorientierte Eigenschaften von weiblichen und männlichen handelnden Personen aktualisieren. Die Genderstereotype spiegelt die gemeinsamen Vorstellungen und Meinungen über den Standard von Männlichkeit und Weiblichkeit wider, die in bestimmter Kultur herausgebildet sind.

In den Romanen «Die Herren von Winterfell» und «Das Erbe von Winterfell» waren 80 genderspezifische Metaphern identifiziert, von denen 25 Frauen und 55 Männern gewidmet sind. Im Roman «A Game of Thrones» waren 79 genderspezifische Metaphern identifiziert, von 
denen 25 Frauen und 54 Männern gewidmet sind. Dieser Unterschied ist mit der Verwendung in der deutschen Übersetzung einer Metapher zur Charakterisierung der männlichen Figur, die im Original nicht angegeben wurde, und zwar mit dem Vergleich der handelnden Person mit mythischem Objekt - Füllhorn, verbunden, wobei es im Original keinen solchen metaphorischen Vergleich gibt.

Die Auswahlkriterien für das untersuchte Material waren 1) ein formal-semantisches Merkmal, d.h. das Vorhandensein einer explizit oder implizit ausgedrückten Gendersemantik, und 2) ein lexikalisch-semantisches Merkmal (Riezanova, 2011: 47-58).

\section{Einteilung genderspezifischer Metaphern nach formal-semantischem Merkmal}

Die im Text zur Charakterisierung männlichen und weiblichen handelnden Personen verwendeten genderspezifischen Metaphern, wurden in die folgenden 4 Gruppen unterteilt: positive Charakteristik von Männern / Frauen, negative Charakteristik von Männern / Frauen, Aussehen von Männern / Frauen, Tätigkeit und Verhalten von Männern / Frauen.

Ziffernmäßig werden diese Gruppen wie folgt klassifiziert:

Metaphern für Charakteristik von Männern:

Positive Eigenschaften von Männern: 2; Negative Eigenschaften von Männern: 15; Aussehen von Männern: 17; Tätigkeit und Verhalten von Männern: 20.

\section{Metaphern für die Charakteristik von Frauen:}

Positive Eigenschaften von Frauen: 3; Negative Eigenschaften von Frauen: 4; Aussehen von Frauen: 2; Tätigkeit und Verhalten von Frauen: 16.

Metaphern für die Charakteristik von Frauen. Für die Beschreibung von Arbeit einer Frau als Spionin vergleicht der Autor sie mit einem Vogel (the bird) und bestimmt damit solche Eigenschaften, die für eine erfolgreiche Selbstverwirklichung in diesem Bereich erforderlich sind: Geschwindigkeit, Effizienz, die Fähigkeit, Informationen. Es sei bemerkt, dass in der Realität der untersuchten Werke Vögel und Krähen die schnellste und zugänglichste Art der Kommunikation sind:

He has informants everywhere. His little birds, he calls them. One of his little birds heard about your visit (Martin, 1996: 213). I Er hat seine Informationen überall. Seine kleinen Vögel nennt er sie. Und seine kleinen Vögel haben von Eurem Besuch erfahren (Martin, 2010: 168).

Eine Frau als Kriegerin ist keine typische Figur in George Martins Romanen. Für ihre Ziele setzen die Heldinnen fast nie körperliche Stärke, aber hauptsächlich List, Macht, Sex ein. In den ersten beiden Romanen haben wir nur wenige Kriegerinnen identifiziert. Mit Hilfe von Femininpronomen passen die Frauen in das männliche Lebensbild hinein. Um ihre aktive Teilnahme an militärischет Handlungen und nicht die letzte Position in der Armee von Rob Stark zu beschreiben, vergleicht der Autor sie mit Snark (the snark), einem Fabelwesen, das den Kindern im Universum von «A Song of Ice and Fire» Furcht einjagd, und ergänzt diese Beschreibung mit folgenden Beiwörtern: hartnäckig, ungeduldig, mutwillig (stubborn, short-tempered, willful / halsstarrig, ungeduldig und eigensinnig).

"Like as not, my sister is marching in your brother's host, her and those daughters of hers, dressed in men's mail. Maege is a hoary old snark, stubborn, short-tempered, and willful» (Martin, 1996: 953). / «Ob es mir gefällt oder nicht: meine Schwester marschiert in der Armee deines Bruders, sie und ihre Töchter, in Männerrüstungen. Maegen ist ein grauer, alter Snark, halsstarrig, ungeduldig und eigensinnig» (Martin, 2010: 340).

Der Text der ersten beiden Romane des Zyklus enthält nur wenige absolut negative weibliche Personenbezeichnungen, und der Vergleich erfolgt hauptsächlich mit Tieren (die Kuh / the cow): 
«You fret too much. Lisa Arryn is a frightened cow». "That frightened cow shared Jon Arryn's bed». (Martin, 1996: 106) / "Du machst dir zu viel Sorgen. Lisa Arryn ist eine schreckhafte Kuh». "Diese schreckhafte Kuh hat mit Jon Arryn das Bett geteilt» (Martin, 2010: 82).

Metaphern für Charakteristik von Männern. Die negativen Personenbezeichnungen von männlichen Figuren im Text der ersten beiden Romane des Zyklus erfolgen häufig durch Vergleich mit weiblichen Figuren in unterschiedlichen Kontexten. Wir sehen also, dass das Vorhandensein oder Fehlen bestimmter Charaktereigenschaften, Mängel oder umgekehrt attraktive Elemente des Aussehens auf Frauen extrapoliert werden, als ob sie ihnen automatisch angeboren wären. Die Kombination von genderorientierten Substantiven mit Geschlechtsmarkern verstärkt die Dichotomie zwischen Männlichkeit und Weiblichkeit und verleiht der Äußerung Bedeutung stereotyper Behauptung. Zum Beispiel erwies sich eine der handelnden Personen in den Werken, Sam Tarle, als kein geschickter Krieger, ein Feigling, außerdem war der junge Mann übergewichtig, wie der Autor im Vergleich mit einer Schweinsau beschreibt (Lady Piggy / die Schweinedame). Solche Aussagen spiegeln die kollektiven Repräsentationen wider, die für Mitglieder einer Gendergruppe gemeinsam sind und auf Stereotypen des Denkens basieren:

"Three of you ought to be sufficient to make Lady Piggy sgueal. All you need do is get past the Bastard» (Martin, 1996: 322). / "Drei von Euch sollten genügen, die kleine Schweinedame zum Quieken zu bringen. Ihr müsst nur den Bastard überwinden» (Martin, 2010: 254).

\section{Einteilung genderspezifischer Metaphern nach lexikalisch-semantischem Merkmal}

Bei der Einteilung von genderspezifischen Metaphern nach lexikalisch-semantischem Merkmal wurden folgende Objektgruppen identifiziert, mit denen handelnde Personen im Text von Romanen verglichen werden: Tiere (Vögel, Reptilien, Insekten), Fabelwesen, abstrakte Begriffe, Artefakte, Körperteile, Material, Mann als weibliche Person, Naturelemente. In Romanen «Die Herren von Winterfell» und «Das Erbe von Winterfell» noch eine Gruppe wurde hervorgehoben - ein mythischer Gegenstand, das im Roman «A Game of Thrones» fehlt. Diese Nominationen werden durch Substantive dargestellt, die in Kombination mit adjektivischer Lexik eine ausdrucksstarke und bewertende Bedeutung erhalten.

Ziffernmäßig werden diese Gruppen wie folgt klassifiziert:

Tiere: 31; Fabelwesen: 5; Abstrakte Begriffe: 1; Artefakte: 5; Körperteile: 2; Material: 1; Naturelemente: 6; Mann als weibliche Person: 3; Mythischer Gegenstand: 1.

Wenn wir Tiere als Vergleichsobjekte im Text von Romanen betrachten, sollte beachtet werden, dass Männer mit dem Hund (the dog), dem Esel (the ass), dem Panther (the panther), dem Wolf (the wolf), dem Bären (the bear), dem Auerochsen (the aurochs), dem Affen (the monkey), dem Löwen (the lion), dem Eichhörnchen (the squirrel), dem Bullen (the bull), der Ratte (the rat), dem Maulwurf (the mole), dem Hirsch (the deer /), dem Hegst (the stallion / der Hegst), dem Igel (the hedgehog), der Spinne (the spider), dem Frosch (the frog) verglichen werden.

Zum Beispiel vergleicht der Autor die Männer aus der königlichen Garde, die sich aus Angst um ihr Leben und Wohlergehen und um jeden Verdacht zu vermeiden den Raum in einer zweideutigen Situation zu verlassen beeilten, mit Hasen (the hares) und Ratten (the rats):

From his vantage point atop of throne, he could see men slipping out the door at the far end of the hall. Hares going to ground, he supposed ... or rats off to nibble the queen's cheese (Martin, 1996: 573). I Von seinem Aussichtspunkt auf dem Thron konnte er sehen, dass Männer aus der Tür auf der anderen Seite des Saales schlichen. Hasen, die in ihren Löchern

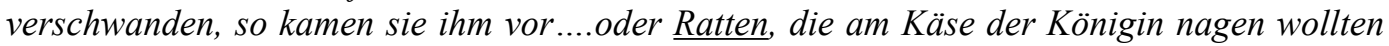
(Martin, 2010: 38). 
Der Mann als Fabelwesen wird in den Texten der Romane mit dem Riesen (the giant), dem Gnomen (the dwarf), dem Teufel (the devil), dem Dämon (the demon), dem Drachen (the dragon) verglichen.

Zum Beispiel wird Tyrion Lannister, ein Zwerg, als ein wirklicher Riese (the giant) bezeichnet, was auf die herausragenden geistigen Fähigkeiten dieses Charakters und seine Fähigkeit hinweist, strategisch und weitsichtig zu denken:

He spoke softly, yet the high officers of the Night's Watch all fell quiet, the better to hear what the ancient had to say. "I think he is a giant come among us, here at the end of the world» (Martin, 1996: 254-255). / Er sprach leise, und die hohen Offiziere der Nachtwache schwiegen, um besser zu hören, was der Greis zu sagen hatte. "Ich denke, er ist ein Riese unter uns, hier am Ende der Welt» (Martin, 2010: 200).

Genderstereotype sind in der Sprache festgelegt, stehen in engem Zusammenhang mit dem Ausdruck der Bewertung und beeinflussen die Bildung von konkreten Erwartungen von Vertretern bestimmten Geschlechts (Кирилина, 2005: 7-32). Die Metapher stellt also kognitiven Mechanismus zur Interpretation der Realität, und die Bewertung wird wiederum als eine der Schlüsselkomponenten der menschlichen kognitiven Aktivität definiert.

25 genderspezifische Metaphern, die Frauen charakterisieren, wurden nach lexikalisch-semantischem Merkmal folgenderweise gruppiert: Tiere (auch Vögel), Fabelwesen, abstrakte Begriffe, Körperteile, Himmelsrichtung, Material. Ziffernmäßig werden diese Gruppen wie folgt klassifiziert:

Tiere: 15; Fabelwesen: 6; Abstrakte Begriffe: 1; Körperteile: 1; Himmelsrichtungen: 1; Material: 1.

Die größte Gruppe bilden genderspezifische Metaphern, die eine Frau durch Vergleich mit einem Tier charakterisieren. Im Text werden die Frauen der Eule (the owl), der Wölfin (die Wölfin), der Henne (the hen), der Maus (the mouse), der Kuh (the cow), dem Wiesel (the weasel), dem Reh (the doe), der Katze (the cat) und der Löwin (the lioness) verglichen.

Zum Beispiel wird eine Frau, deren Verhalten im Bett eine der handelnden Personen befriedigt, als das Wiesel bezeichnet (the weasel):

"Sweet Kyra», he said with a laugh. "She squirms like a weasel in bed, but say a word to her on the street, and blushes pink as a maid» (Martin, 1996: 488). / "Die süße Kyra», sagte er lachend. "Im Bett ist sie ein Wiesel, doch sagt man auf der Straße auch nur ein Wort zu ihr, errötet sie wie eine Jungfer» (Martin, 2010: 386).

Der Mangel an herausragenden geistigen Fähigkeiten bei einer Frau wird im Text der Romane durch einen Vergleich mit einem Huhn reflektiert (the hen):

Old Nan had cacckled like a hen when Bran told her that, and confessed that Hodor's real name was Walder (Martin, 1996: 299). / Die Alte Nan hatte wie eine Henne gegackert, als Bran ihr das erzählte, und ihm anvertraut, dass Hodor in Wahrheit Walder hieße (Martin, 2010: 235).

\section{Fazit}

Die Untersuchung der Sprache von Romanen "A Game of Thrones", "Die Herren von Winterfell» und «Das Erbe von Winterfell” gewährte die Möglichkeit, Gendermetaphern, die Männer und Frauen als handelnde Personen in Werken charakterisieren, zu identifizieren und zu gruppieren, sowie die Verwendung metaphorischer Nominationen für Männer und Frauen in der Originalsprache und in der deutschen Übersetzung zu vergleichen. Die Struktur einer auf Männer ausgerichteten Gendermetapher ist gekennzeichnet durch solche Merkmale wie Stärke, Beharrlichkeit, Grausamkeit, Kampfbereitschaft, aktive Lebensposition, Rücksichtslosigkeit, 
List, Selbstvertrauen und Unbeständigkeit politischer Überzeugungen. In der Struktur der Gendermetaphern, die Frauen charakterisieren, können solche Merkmale, wie Klugheit, Leidenschaft, List und Beharrlichkeit abgesondert werden. Gendermetaphern dienen dazu, das Bild einer Frau zu schaffen, wobei negative und positive Charakter- und Verhaltensmerkmale, weibliche Eigenschaften, die für Männer axiologisch bedeutsam sind, sowie die Verbindung der weiblichen Entität mit der Natur ausgedrückt werden. Die Frauen werden zugleich spöttisch oder herablassend aus der Perspektive von Männern charakterisiert. Die metaphorische Verkörperung des Männerbildes verwirklicht die negativen und positiven Eigenschaften von Charakter und Verhalten sowie die Besonderheiten des Lebensstils und der Wahrnehmung der umgebenden Realität.

\section{Literaturverzeichnis}

Andriichenko, Yu. V. (2011) Henderna markovanist odynyts movy [Gender markedness ma language units]. Kyiv. Kyivskyi natsionalnyi universytet imeni Tarasa Shevchenka. [in Ukrainian] Belova, T. M. Elektronnyi resurs. Rezhym dostupu: [Electronic resource. Access mode] http://cheloveknauka.com/gendernaya-metafora-kak-otrazhenie-kulturnogo-kontsepta-maskulinnost-vo-frantsuzskom-yazyke\#ixzz5eaMfduwL

de Lauretis, T. (1998) Amerikanskiy Freyd. Gendernyie issledovaniya. [American Freud. Gender studies.]. SPb. Aleteiia. [in Russian]

Kirilina, A. V. (2005) Gendernyie issledovaniya v lingvisticheskih distsiplinah. Gender $i$ yazyik. [Gender studies in linguistic disciplines. Gender and language.]. M.: Yazyki slavyanskoj kul'tury. [in Russian]

Kostikova, I. V. (2005) Vvedenie v gendernyie issledovaniya. [Introduction to gender studies]. M.: Aspekt Press. [in Russian]

Pushkareva, N. L. (1999) Zhenschina. Gender. Kultura. [Woman. Gender. Culture.] M.: MTsGI. [in Russian]

Rezanova, Z. I. (2011) Gendernaya metafora: tipologiya, leksikograficheskaya interpretatsiya, kontekstnaya reprezentatsiya. [Gender metaphor: typology, lexicographical interpretation, contextual representation.]. Tomsk: izdatel'stvo Tomskogo nacional'nogo universiteta [in Russian]

Semashko, T. M. (2010) Henderna linhvistyka v systemi suchasnoi movoznavchoi nauky. [Gender linguistics in the system of modern linguistic science.]. Mariupol, "Redaktsiino-vydavnychyi viddil MDU» [in Ukrainian]

Slovar gendernyih terminov / pod red. A. A. Denisovoy. Rezhim dostupa [Dictionary of Gender Terms /Ed. A. A. Denisova. Access mode]: http://www.owl.ru/gender/alphabet.htm

Smit, S. (1997) Postmodernizm i sotsialnaya istoriya na zapade: problemy i perspektivy. Voprosy istorii. [Postmodernism and social history in the West: problems and prospects. Problems of history]. M.: RAN [in Russian]

Teoriya metaforyi. (1990) Sbornik. Pod red. Arutyunovoy N. D., Zhurinskoy M. A. [Theory of Metaphor. Collection. Ed. Arutyuna N. D., Zhurinskaya M. A.] M.: Progress. [in Russian] George R. R. Martin. (2010) Das Lied von Eis und Feuer. Die Herren von Winterfell. Ins Deutsch übertragen von Jörn Ingwersen. Blanvalet. München.

George R. R. Martin. (2010) Das Lied von Eis und Feuer. Das Erbe von Winterfell. Ins Deutsch übertragen von Jörn Ingwersen. Blanvalet. München.

George R. R. Martin. (1996) A Game of Thrones, A Song of Ice and Fire: Book One. Bentam Books. New York. 